\title{
Nueva introducción de Aedes vittatus (Diptera: Culicidae) en la región centro- oriental de Cuba: caracterización ecológica y relevancia médica
}

Ireté Díaz Martínez1, Lorenzo Diéguez Fernández ${ }^{\text {* }}$

Beatriz Santana Águila1, Enrique Marcelo Atiénzar de la Paz ${ }^{1}$

Daineris Ruiz Domínguez ${ }^{1}$

Pedro María Alarcón-Elbal²

\footnotetext{
1.Centro Provincial de Higiene, Epidemiología y Microbiología (CPHEM) de Camagüey, Cuba. *Autor para correspondencia: lfdieguez.cmw@infomed. sld.cu; lorenzodieguez95@gmail.com.

2.Universidad Agroforestal Fernando Arturo de Meriño (UAFAM), Jarabacoa, República Dominicana.
}

\section{Resumen}

Aedes vittatus (Diptera: Culicidae) posee una amplia distribución en el Viejo Mundo, que se ha ampliado recientemente al continente americano. Este mosquito presenta una marcada preferencia por hábitats cercanos a las poblaciones humanas y se relaciona con la transmisión de arbovirus de importancia médica. A principios de diciembre de 2020, después de una inspección rutinaria en una localidad rural de Camagüey, Cuba, se capturaron estados inmaduros de culícidos en diferentes tipos de reservorios. Tras la maduración de las larvas y la eclosión de las pupas capturadas, se identificaron un total de 34 larvas y nueve adultos pertenecientes a dicha especie, entre otras de importancia médica. Esta es la primera detección de Ae. vittatus en Cuba fuera de la Base Naval de la Bahía de Guantánamo, y la tercera en las Américas. Es necesario continuar profundizando en la bioecología y distribución de esta potencial especie invasora, así como determinar el impacto futuro que tendrá la presencia de este mosquito en Cuba.

Palabras clave: Aedes vittatus; Especies de mosquitos invasores; Arbovirus; Encuesta entomológica; Caribe insular, Cuba.

\section{Abstract}

Aedes vittatus (Diptera: Culicidae) has a wide distribution in the Old World, and it has recently been identified on the American continent. This mosquito has a marked preference for habitats close to human populations, and it is responsible for the transmission of arboviruses of medical importance. In early December 2020, after a routine inspection in a rural locality of Camagüey, Cuba, immature forms of culicids were captured in different types of reservoirs. After the development of the larvae and hatching of the captured pupae, 34 larvae and nine adults belonging to this species were identified, among other species of medical importance. This is the first detection of Ae. vittatus in Cuba outside the Guantanamo Bay Naval Base, and the third report in the Americas. It is necessary to deepen our understanding of the bioecology and distribution of this potential invasive species and determine the future impact of the presence of this mosquito in Cuba.

Keywords: Aedes vittatus; Invasive mosquito species; Arbovirus; Entomological survey; Insular Caribbean, Cuba. 


\section{Introducción}

La dispersión mundial de los mosquitos (Diptera: Culicidae) se relaciona generalmente con actividades humanas como el comercio y el transporte (1); tales son los casos de los mosquitos invasores Aedes (Stegomyia) aegypti (Linnaeus, 1762), Aedes (Hulecoeteomyia) japonicus japonicus (Theobald, 1901) y Aedes (Stegomyia) albopictus (Skuse, 1894) (2, 3). La rápida dispersión mundial de este último, acaecida en las últimas décadas, es el mejor ejemplo de introducciones exitosas en áreas no endémicas de arbovirosis como el dengue y el chikungunya. La incursión de esta especie sinantrópica desde el sudeste asiático a través de huevos en neumáticos usados (4), ha provocado la aparición de brotes de estas enfermedades en los nuevos territorios colonizados, como en algunos países del sur de Europa (5).

La actual situación epidemiológica respecto a varias enfermedades transmitidas por vectores, ha incrementado la atención que la comunidad científica dirige hacia los mosquitos (6); por lo que hay una imperiosa necesidad de desarrollar investigaciones en el campo taxonómico, que permitan conocer la presencia y distribución de las diferentes especies de culícidos, y entre éstos sobre todo los vectores, en un lugar determinado. En los casos de Ae. aegypti y $A$ e. albopictus, se han diseñado e implementado estrategias de vigilancia y control en Camagüey, ubicada en la región centro-oriental de Cuba, dada su amplia dispersión en la provincia. Dichas acciones están dirigidas a reducir los daños que provocan estas especies a la salud humana, entre lo que destaca la importancia de contar con el inventario de los culícidos y su actualizada geodistribución, para ejecutar acciones antivectoriales más acertadas y eficientes.

En el presente artículo se describe por primera vez la presencia de la especie Aedes (Fredwardsius) vittatus (Bigot, 1861) en la provincia de Camagüey, un mosquito exótico que interviene en la transmisión biológica de importantes arbovirosis (7), relacionándola además con sus sitios de cría en condiciones naturales.

\section{Material y métodos}

El 3 de diciembre de 2020 se realizó una encuesta entomológica en el universo rural de la localidad Isabel Hortensia, ubicada a $2 \mathrm{~km}$ de la Circunvalación Sur carretera destino a Vertientes, en el camino Buey de

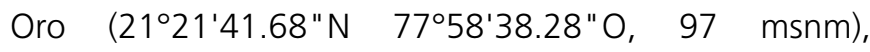

perteneciente al municipio de Camagüey, provincia de Camagüey, y atendida por el Departamento de Control de Vectores del Área de Salud del Policlínico Previsora. Dicha encuesta, ejecutada en el marco de una inspección rutinaria en el municipio cabecera, incluyó el muestreo de todos los criaderos naturales en un radio aproximado de $1 \mathrm{~km}$ alrededor del asentamiento humano, así como todos los depósitos artificiales presentes dentro y en los alrededores de las viviendas. Esta localidad se caracteriza por tener extensas fincas que se dedican a labores agrícolas y a la cría de animales domésticos, donde se encuentran abundantes cuerpos de agua, como lagunas y charcas estacionales.

La clasificación de los depósitos se realizó atendiendo a la utilidad que le confieren las familias a los mismos: permanentes y útiles (PU), los que siempre tienen agua para diversos usos; no permanentes (NP), los que no la tienen y, generalmente, están abandonados en áreas exteriores de las viviendas, como el patio y el jardín; y los naturales (N) (8). En cada criadero se recolectó la mayor cantidad posible de larvas y/o pupas, con ayuda de pipetas Pasteur, bandejas plásticas y botes herméticos.

Una vez en el insectario del Departamento de Control de Vectores del Centro Provincial de Higiene, Epidemiología y Microbiología de Camagüey, las larvas se introdujeron en botes de eclosión para su maduración hasta el estadio larvario $L_{4}$, tras lo cual se procedió a su fijación en alcohol al $70 \%$, previa muerte por baño de agua caliente a $60^{\circ} \mathrm{C}$. Las pupas se dejaron eclosionar para realizar la clasificación de los adultos, que se sacrificaron con frío y se conservaron en fresco. El material biológico fue clasificado utilizando claves taxonómicas $(9,10,11$, 12).

\section{Resultados}

En la Tabla 1 se observa que se capturaron ocho especies de culícidos en siete tipos de depósitos, todos ellos localizados en el exterior de las viviendas, siendo estas: Ae. vittatus, Ae. aegypti, Ae. albopictus, Culex coronator (Dyar \& Knab, 1906), Culex nigripalpus Theobald, 1901, Culex quinquefasciatus Say, 1826, Culiseta inornata (Williston, 1893) y Anopheles albimanus Wiedemann, 1820. La presencia de Ae. vittatus se reportó en tres tipos de criaderos (42.85\%), mientras que, de los 14 depósitos totales con larvas, en cuatro se detectó dicha especie $(28.57 \%)$. Se destacaron los neumáticos usados con el $50.00 \%$ de la positividad para este culícido. En 
total se capturaron 147 larvas, destacándose en orden Ae. albopictus (41.49\%), Ae. vittatus (23.12 \%) y CX. quinquefasciatus (14.96\%). El mayor aporte de pupas correspondió a Ae. vittatus (60.00\%). Esta especie se asoció a otras de marcado seguimiento médico como Ae. aegypti y Ae. albopictus, criando tanto en depósitos artificiales PU (un abrevadero de cemento y un tanque bajo) (50.00\%), como en NP (dos neumáticos usados) (50.00\%), respectivamente. En la Tabla 1, se relaciona el resto de los depósitos en los que no se capturó Ae. vittatus, pero que constituyeron sitios de cría potenciales y en los que se capturaron especies con las cuales se asoció, la mayoría de relevancia vectorial.

De las muestras de estados preimaginales colocadas en el insectario se obtuvieron seis machos y tres hembras de Ae. vittatus (Figura 1), cuyo carácter sistemático más relevante es su patrón de escamas en la región dorsal del tórax, formado por tres pares de manchas blancas redondeadas situadas a lo largo de la línea acrostical que le dan un aspecto típicamente moteado.

\section{Discusión}

El reciente primer reporte de $A$ e. vittatus en la región de las Américas en República Dominicana (13), sumado al posterior en la Base Naval de la Bahía de Guantánamo (14), abre muchas incógnitas desde el punto de vista epidemiológico, sobre todo por conocer cuál puede ser el rol de esta especie en los brotes de arbovirus que azotan el Caribe insular. Tanto Ae. aegypti como Ae. albopictus han podido mantener epidemias exóticas del virus chikungunya en las Américas y el Caribe; en este sentido, Ae. vittatus se ha demostrado como vector de dichos virus, a los que se agregan el del dengue y la fiebre amarilla $(7,15)$, lo que eleva el riesgo epidemiológico, si además se le suma la cohabitación con Ae. aegypti y Ae. albopictus, como es el caso.

Aedes vittatus se distribuye en el Viejo Mundo a través del continente africano, Asia tropical y sur de Europa, mostrando un comportamiento intra y peridoméstico, donde suele colonizar depósitos naturales como oquedades de piedras y agujeros en árboles ( 7 , 16), y favorecido, además, por una probada plasticidad ecológica que le permite colonizar recipientes artificiales (11). La tolerancia a la desecación de sus huevos, fenómeno presente en muchas especies del género Aedes, ha sido una de las claves de su éxitosa distribución mundial a través del comercio, muchas veces indavertida hasta que sus poblaciones, ya establecidas, hacen que su control sea de muy difícil consecución.

Los neumáticos usados parecen tener un papel protagónico dentro de los recipientes artificiales que coloniza este aedino en Camagüey, lo que fortalece la idea de que esta especie pudo arribar al continente americano a través del comercio de estos productos, en concreto desde el subcontinente indio $(13,14)$. De hecho, este tipo de residuos sólidos de caucho se ha ratificado recientemente como uno de los recipientes clave que albergan especies de mosquitos de interés médico en el Caribe insular (17).

El haber capturado a esta especie en territorio cubano fuera de la Base Naval de Guantánamo, a más de $400 \mathrm{~km}$ de distancia, en una localidad rural a $5 \mathrm{~km}$ de la capital provincial y además ubicada en la región centro-oriental de la isla, hace sospechar que su invasión a través del país ha podido comenzar hace algunos años, y que es posible que pueda estar presente en otros países antillanos, aunque todavía permanezca sin detectar. Resultó interesante observar que el entorno donde se hicieron las capturas larvarias es muy similar al de República Dominicana (13), pues en ambos países se encontró en localidades bastante rurales con campos abiertos, zonas cenagosas y presencia de animales domésticos. Esto sugiere un probable cambio en el escenario epidemiológico de las arbovirosis, ya que la especie al parecer suele preferir y colonizar ambientes y criaderos naturales (18), a pesar de que en dicho estudio se encontró solo en recipientes artificiales, entornos muy diferentes a los utilizados por las dos especies de mayor relevancia vectorial, Ae. aegypti y Ae. albopictus, cuyas abundancias son siempre notables en ambientes urbanos, sobre todo en el caso de la primera.

En conclusión, estos resultados tienen implicaciones significativas para el ecosistema y la salud humana. Sin duda, esta introducción no intencional puede representar un cambio en el escenario epidemiológico de enfermedades endémicas como el dengue, añadiendo, si cabe, un grado más de complejidad a su control. Por tanto, se requiere conocer en profundidad la bioecología de esta especie y los lugares en donde este potencial invasor se encuentra establecido, así como determinar el futuro impacto que tendrá la presencia de esta nueva especie de culícido exótico en Cuba. 


\section{References}

1. Schaffner $F \&$ Mathis $A$. Dengue and dengue vectors in the WHO European region: past, present, and scenarios for the future. Lancet Infect Dis. 2014;14:1271-1280.

2. Kaufman MG \& Fonseca DM. Invasion biology of Aedes japonicus japonicus (Diptera: Culicidae). Annu Rev Entomol. 2014;59:31-49.

3. Paupy C, Delatte H, Bagny L, Corbel V \& Fontenille D. Aedes albopictus, an arbovirus vector: from the darkness to the light. Microbes Infect. 2009;11(14-15):1177-1185.

4. Knudsen $A B$. Global distribution and continuing spread of Aedes albopictus. Parassitologia. 1995;37:91-97.

5. Gossner CM, Ducheyne E \& Schaffner F. Increased risk for autochthonous vector-borne infections transmitted by Aedes albopictus in continental Europe. Euro Surveill. 2018;23(24):pii=1800268.

6. Diéguez-Fernández L, Díaz-Martínez I, Santana-Aguila B, Atienzar de la Paz EM, Ruiz Domínguez D, Prada Noy Y, lannacone J, et al. Culex (Culex) coronator (Dyar \& Knab) (Arthropoda: Diptera: Culicidae) in Camagüey, Cuba. Neotrop Helminthol. 2020;14(1):105-109.

7. Sudeep $A B$ \& Shil P. Aedes vittatus (Bigot) mosquito: An emerging threat to public health. J Vector Borne Dis. 2017;54(4):295-300.

8. Diéguez Fernández L, Cabrera Fernández SM, Prada Noy $Y$, Cruz Pineda C \& Rodríguez de la Vega R. Aedes (St.) aegypti en tanques bajos y sus implicaciones para el control del dengue en Camagüey. Rev Cubana Med Trop. 2010;62(2):93-97.

9. ServiceMW. Studies on thebiologyand taxonomy of Aedes (Stegomya) vittatus (Bigot) (Diptera: Culicidae) in northern Nigeria. Trans R Soc Entomol Soc Lond. 1970;122:101-143.

10. Huang YM. Medical entomology studies-VIII. Notes on the taxonomic status of Aedes vittatus (Diptera: Culicidae). Contrib Am Entomol Inst. 1977;14:113-132.
11. Schaffner F, Angel G, Geoffroy B, Hervy JP, Rhaiem A \& Brunhes J. Les moustiques d'Europe: logiciel d'identification et d'enseignement. CD-ROM. Paris: IRD Editions. 2001.

12. González Broche R. Culícidos de Cuba. La Habana: Ed. Científico Técnica. 2006. 183 pp.

13. Alarcón-Elbal PM, Rodríguez-Sosa MA, Newman BC \& Sutton WB. The First Record of Aedes vittatus (Diptera: Culicidae) in the Dominican Republic: Public Health Implications of a Potential Invasive Mosquito Species in the Americas. J Med Entomol. 2020;57(6):2016-2021.

14. Pagac $B B$, Spring $A R$, Stawicki JR, Dinh $T L$, Lura $T$, Kavanaugh MD, Pecor $D B$, et al. Incursion and establishment of the Old World arbovirus vector Aedes (Fredwardsius) vittatus (Bigot, 1861) in the Americas. Acta Trop. 2021;213:105739.

15. Diagne CT, Diallo D, Faye O, Ba Y, Faye O, Gaye A, Dia I, et al. Potential of selected Senegalese Aedes spp. mosquitoes (Diptera: Culicidae) to transmit Zika virus. BMC Infect Dis. 2015;15:492.

16. Diallo D, Sall AA, Diagne CT, Faye O, Faye O, Ba Y, Hanley $K A$, etal.Zikavirus emergence in mosquitoes in southeastern Senegal, 2011. PLoS One. 2014;9(10):e109442.

17. González MA, Rodríguez Sosa MA, Vásquez Bautista YE, Rosario EC, Durán Tiburcio JC \& Alarcón-Elbal PM. A survey of tire-breeding mosquitoes (Diptera: Culicidae) in the Dominican Republic: considerations about a pressing issue. Biomédica. 2020;40(3):507-515.

18. DialloD, DiagneCT, HanleyKA, Sall AA, BuenemannM, Ba Y, Dial, etal. Larvalecology of mosquitoes in sylvaticarbovirus foci in southeastern Senegal. Parasit Vectors. 2012;5:286. 
Tabla 1. Relación de los depósitos positivos a culícidos en la localidad rural Isabel Hortensia, Camagüey, en diciembre de 2020.

\begin{tabular}{|c|c|c|c|c|c|}
\hline Depósitos & $\begin{array}{c}\text { Total } \\
\text { depósitos positivos } \\
\text { culícidos }\end{array}$ & $\begin{array}{c}\text { Total } \\
\text { depósitos positivos } \\
\text { Ae. vittatus }\end{array}$ & $\begin{array}{l}\text { Total } \\
\text { ejemplares } \\
\text { Ae. vittatus }\end{array}$ & $\begin{array}{l}\text { Otras especies } \\
\text { asociadas }\end{array}$ & $\begin{array}{c}\text { Total } \\
\text { ejemplares }\end{array}$ \\
\hline $\begin{array}{l}\text { Abrevadero (PU) } \\
\text { Tanque bajo (PU) }\end{array}$ & $\begin{array}{l}1 \\
3\end{array}$ & $\begin{array}{l}1 \\
1\end{array}$ & $\begin{array}{c}16 \mathrm{~L}, 5 \mathrm{P} \\
2 \mathrm{~L}\end{array}$ & $\begin{array}{c}\text { Cx. coronator } \\
\text { Ae. aegypti } \\
\text { Cx. quinquefasciatus } \\
\text { Cs. inornata }\end{array}$ & $\begin{array}{l}2 \mathrm{~L} \\
4 \mathrm{~L} \\
9 \mathrm{~L} \\
1 \mathrm{~L}\end{array}$ \\
\hline $\begin{array}{c}\text { Neumático usado } \\
\text { (NP) }\end{array}$ & 6 & 2 & $16 \mathrm{~L}, 4 \mathrm{P}$ & $\begin{array}{c}\text { Ae. aegypti } \\
\text { Ae. albopictus } \\
\text { Cx. quinquefasciatus }\end{array}$ & $\begin{array}{c}6 \mathrm{~L} \\
54 \mathrm{~L}, 5 \mathrm{P} \\
9 \mathrm{~L}\end{array}$ \\
\hline Lechera (NP) & 1 & - & - & Ae. aegypti & $5 \mathrm{~L}$ \\
\hline Comedero (PU) & 1 & - & - & $\begin{array}{l}\text { Cx. coronator } \\
\text { Cx. nigripalpus }\end{array}$ & $\begin{array}{c}4 \mathrm{~L}, 1 \mathrm{P} \\
2 \mathrm{~L}\end{array}$ \\
\hline Laguna (N) & 1 & - & - & $\begin{array}{c}\text { Cx. coronator } \\
\text { Cx. nigripalpus } \\
\text { Cx. quinquefasciatus } \\
\text { An. albimanus }\end{array}$ & $\begin{array}{l}2 \mathrm{~L} \\
2 \mathrm{~L} \\
1 \mathrm{~L} \\
2 \mathrm{~L}\end{array}$ \\
\hline & 14 & 4 & $34 \mathrm{~L}, 9 \mathrm{P}$ & & $113 \mathrm{~L}, 6 \mathrm{P}$ \\
\hline
\end{tabular}

Leyenda: PU, depósito permanente y útil; NP, depósito no permanente; N, depósito natural; L, larvas; P, pupas. 
Figura 1. Vista general de una hembra de Aedes vittatus emergida de los botes de eclosión.

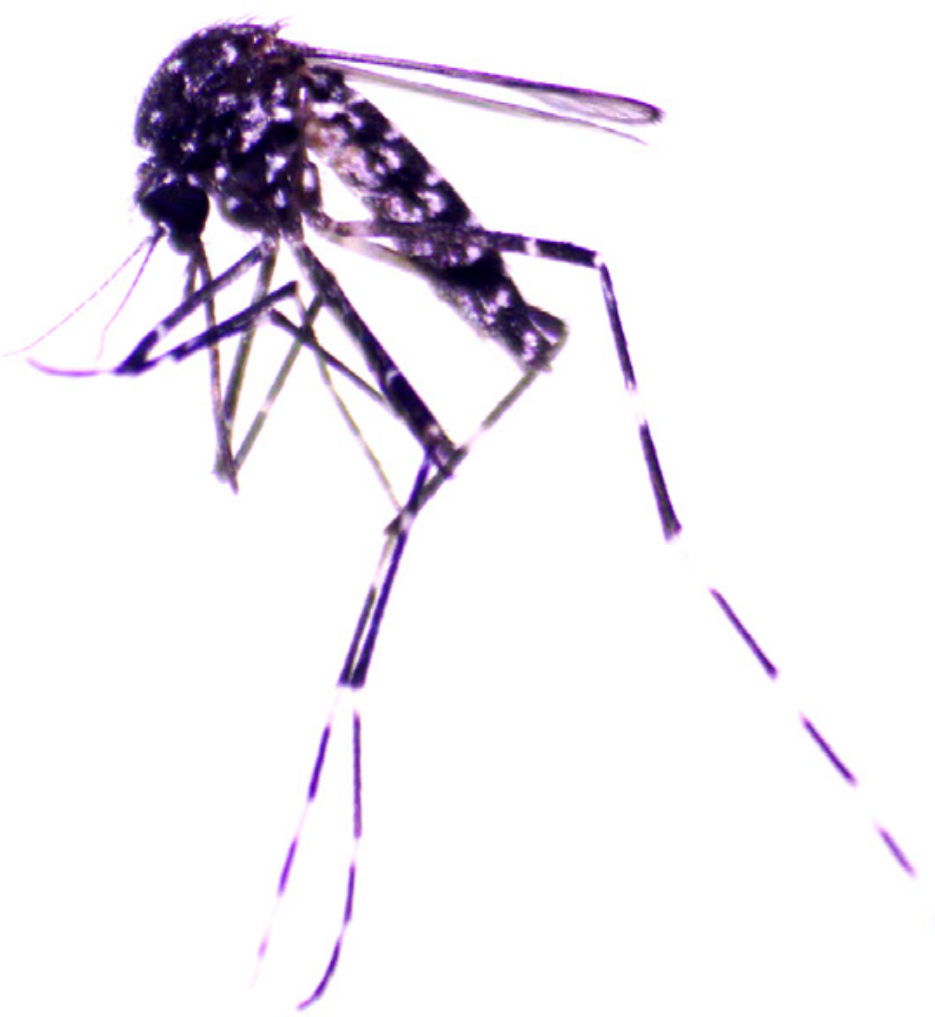

